\title{
Pretreatment Method for Immunoassay of Polychlorinated Biphenyls in Transformer Oil Using Multilayer Capillary Column and Microfluidic Liquid-Liquid Partitioning
}

\author{
Arata AOTA, ${ }^{* \dagger}$ Yasumoto Date, $* * *$ Shingo TeraKado,* and Naoya OHMURA* \\ *Environmental Science Research Laboratory, Central Research Institute of Electric Power Industry, \\ 1646 Abiko, Abiko, Chiba 270-1194, Japan \\ **Graduate School of Environmental Studies, Tohoku University, 6-6-11 Aramaki, Aoba, Sendai, \\ Miyagi 980-8579, Japan
}

\begin{abstract}
Polychlorinated biphenyls (PCBs) are persistent organic pollutants that are present in the insulating oil inside a large number of transformers. To aid in eliminating PCB-contaminated transformers, PCBs in oil need to be measured using a rapid and cost-effective analytical method. We previously reported a pretreatment method for the immunoassay of PCBs in oil using a large-scale multilayer column and a microchip with multiple microrecesses, which permitted concentrated solvent extraction. In this paper, we report on a more rapid and facile pretreatment method, without an evaporation process, by improving the column and the microchip. In a miniaturized column, the decomposition and separation of oil were completed in $2 \mathrm{~min}$. PCBs can be eluted from the capillary column at concentrations seven-times higher than those from the previous column. The total volume of the microrecesses was increased by improving the microrecess structure, the enabling extraction of four-times the amount of PCBs achieved with the previous system. By interfacing the capillary column with the improved microchip, PCBs in the eluate from the column were extracted into dimethyl sulfoxide in microrecesses with high enrichment and without the need for evaporation. Pretreatment was completed within 20 min. The pretreated oil was analyzed using a flow-based kinetic exclusion immunoassay. The limit of detection of PCBs in oil was $0.15 \mathrm{mg} \mathrm{kg}^{-1}$, which satisfies the criterion set in Japan of $0.5 \mathrm{mg} \mathrm{kg}^{-1}$.
\end{abstract}

(Received October 12, 2012; Accepted February 15, 2013; Published April 10, 2013)

\section{Introduction}

Polychlorinated biphenyls (PCBs) were used in various industrial fields, particularly for capacitors and transformers, because of their high chemical and thermal stabilities, and low conductivity. ${ }^{1}$ However, in the 1970 s, their toxicity and environmental persistence became apparent. ${ }^{2}$ Consequently, their production and use were banned in many countries. In May 2004, PCBs were banned worldwide by the Stockholm Convention on Persistent Organic Pollutants (POPs). ${ }^{3}$ However, PCBs are still being used as insulating oils because devices such as transformers have lifetimes exceeding several decades. Furthermore, in 2003, the Japanese government reported unintended PCB contamination in transformers produced after 1973, when Japan banned the production and use of PCBs. A large number of transformers are therefore thought to be contaminated. Consequently, the Japanese government has recommended that every transformer be checked for $\mathrm{PCB}$ contamination to identify PCB-contaminated transformers and safely dispose of them when they are removed from service.

Gas chromatography coupled with either mass spectrometry or electron capture detection is generally used for the determination of PCBs in transformer oil. ${ }^{4-7}$ Although their high

$\dagger$ To whom correspondence should be addressed.

E-mail: aota@criepi.denken.or.jp sensitivity and high accuracy are attractive, these methods are time consuming and costly. Immunoassays for rapid and low-cost screening of PCBs in oil have been proposed. ${ }^{8-11}$ By using the selectivity of a specific antibody for PCBs, the pretreatment procedure can be simplified. We previously developed a pretreatment method and immunoassay for PCBs in mineral oil. ${ }^{8,9}$ The pretreatment method consisted of a large-scale multilayer silica-gel column process, hexane evaporation, and dimethyl sulfoxide (DMSO) partitioning. The immunoassay was based on a flow-based immunoassay with absorbance measurements using a handheld readout instrument. Its usefulness as a screening method is evident from receiver-operating characteristic plots of 500 real oil samples. ${ }^{12}$ These systems have been approved by the Japanese Ministry of the Environment for rapid low-cost screening and are now commercially available. ${ }^{13}$ However, it is difficult to use the present system for on-site screening because of the large evaporation equipment required. The evaporation process for the enrichment of PCBs therefore needs to be eliminated. If less-diluted column operation and concentrated DMSO partitioning were achieved, the evaporation process could be eliminated.

We previously demonstrated an immunoassay of PCBs in oil using microfluidic concentrated DMSO partitioning in multiple circle-like microrecesses aligned along a microchannel. ${ }^{14}$ First, a $295-\mu \mathrm{L}$ aliquot of PCB-contaminated oil was treated with a large-scale multilayer column. The eluate, with $10 \mathrm{~mL}$ of 
hexane, was evaporated. The evaporated eluate was flowed into a microchip with multiple microrecesses. PCBs in the evaporated eluate were extracted into DMSO confined in the microrecesses. DMSO in the microrecesses was washed out by dissolving in $1 \mathrm{~mL}$ of phosphate-buffered saline (PBS). The obtained solution was used for a flow-based immunoassay. Although the previous method decreased the space required for pretreatment and eliminated the use of centrifugation, an evaporation process was required. In order to eliminate the evaporation process, the multilayer column and the microchip need to be improved. A large-scale column cannot make effective use of the reagents because the oil is contacted with only a portion of the reagent. Furthermore, a large volume of hexane is required for eluting the PCBs, because of the diffusion of PCBs in the large-scale column. The PCBs in the eluate are therefore highly diluted. Miniaturization of the column may achieve highly efficient contact with the reagent and less dilution of the PCBs. Furthermore, reductions of the amounts of reagents and oil samples may be achieved. A microchip with multiple microrecesses enables concentrated DMSO partitioning. When the total volume of DMSO confined in the microrecesses is increased, larger amounts of PCBs are obtained in the DMSO. The increase in the total volume of the microrecesses influences the sensitivity of the flow-based immunoassay. By using a continuous process combining the improved column and improved microchip for DMSO partitioning, the pretreatment could be simplified and made more cost-effective. Furthermore, the pretreatment time is expected to be shortened because of eliminating the evaporation process. The total pretreatment time for the previous method was more than $1 \mathrm{~h}$, which included preparing the evaporation instrument.

In this paper, we report on an improved pretreatment method using a multilayer column and microfluidic DMSO partitioning. An improved multilayer column using a capillary capable of highly efficient oil sulfonation and separation was developed and evaluated. An improved microchip used to increase the amount of PCBs in DMSO was evaluated. By interfacing the multilayer capillary column with the improved microchip, a rapid, continuous pretreatment process was achieved, which does not require an evaporation process and requires only a small amount of reagent.

\section{Experimental}

\section{Reagents and chemicals}

Commercial mixtures of PCBs (Kanechlor [KC] 300, 400, 500 , and $600 ; 1: 1: 1: 1$ mixture) were purchased from GL Sciences Inc. (Tokyo, Japan). DMSO (Catalog No. 346-03615) was purchased from Dojindo Laboratories (Kumamoto, Japan). A blocking reagent, N101, was purchased from NOF Corp. (Tokyo, Japan). Acetone and hexane were purchased from Wako Pure Chemical Industries, Ltd. (Osaka, Japan). PBS (consisting of $137 \mathrm{mM}$ sodium chloride, $3 \mathrm{mM}$ potassium chloride, $20 \mathrm{mM}$ disodium hydrogen phosphate, $1.5 \mathrm{mM}$ potassium dihydrogen phosphate, and $1.5 \mathrm{mM}$ sodium azide; $\mathrm{pH}$ 7.4) was prepared in-house. PCB-free insulating oil for electric transformers was purchased from Matsumura Oil Co., Ltd. (Barrel Trans M; Osaka, Japan). Anhydrous sodium sulfate, oleum-impregnated silica gel, and aminopropyl silica gel were purchased from Sumika Chemical Analysis Service, Ltd. (Osaka, Japan). A positive photoresist (S-1818) was purchased from Nippon Kayaku Co., Ltd. (Tokyo, Japan). Fluorescent polystyrene microparticles (F8823) were purchased from Life Technologies Japan, Ltd. (Tokyo, Japan). A monoclonal anti-
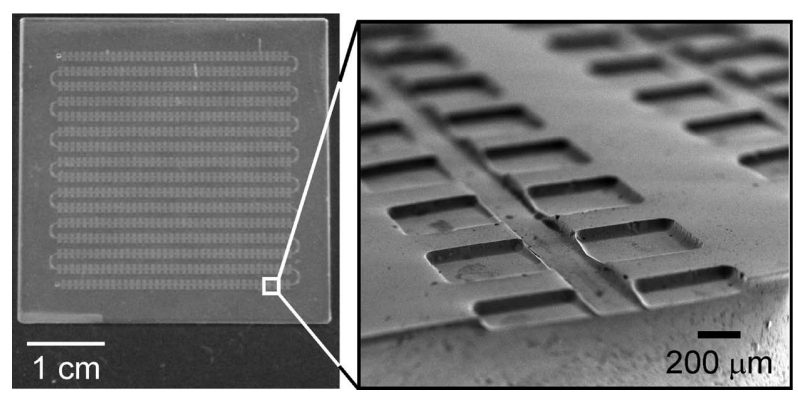

Fig. 1 Photograph and scanning electron microscopy image of the microchip. The main microchannel has a width of $260 \mu \mathrm{m}$, a depth of $50 \mu \mathrm{m}$, and a length of $610 \mathrm{~mm}$. Each of the 1212 rectangular microrecesses has a width of $520 \mu \mathrm{m}$ and a depth of $50 \mu \mathrm{m}$. Since the volume of each microrecess was estimated to be $12.2 \mathrm{~nL}$, the total volume of the microrecesses was approximately $14.8 \mu \mathrm{L}$.

PCB antibody (K2A) was purchased from Kyoto Electrics Manufacturing Co., Ltd. (Kyoto, Japan). Cy-5-labeled F(ab')2 fragment goat anti-mouse $\operatorname{IgG}(\mathrm{H} \& \mathrm{~L})$ was purchased from Jackson ImmunoResearch Laboratories Inc. (West Grove, PA).

\section{Microchip}

A photolithographic wet-etching method was used to fabricate microchannels on a glass substrate, as described in our previous paper. $^{14}$ The fabricated microchannels were covered with another substrate with drilled $0.5-\mathrm{mm}$ inlet and outlet holes, using a thermal bonding method. Figure 1 shows a photograph and scanning electron microscopy image of the microchip, which had a main microchannel $(L 610 \mathrm{~mm} \times W 260 \mu \mathrm{m} \times$ $D 50 \mu \mathrm{m}$ ) and 1212 rectangular microrecesses ( $L 520 \mu \mathrm{m} \times$ $W 520 \mu \mathrm{m} \times D 50 \mu \mathrm{m})$. The volume of the main microchannel was estimated to be $7.3 \mu \mathrm{L}$. Considering the rounded structures of the microrecesses, resulting from fabrication using a wet-etching method, the volume of each microrecess was estimated to be $12.2 \mathrm{~nL}$, which led to a total volume of the microrecesses of approximately $14.8 \mu \mathrm{L}$. Since the total volume of the circle-like microrecesses of the previous microchip was $3.5 \mu \mathrm{L}$, a four-times larger volume could be obtained in the improved microchip. The microchip was used for multiple experiments and was washed with acetone after each use.

\section{Capillary multilayer column}

PCB cleanup was performed using a capillary multilayer column. Anhydrous sodium sulfate, oleum-impregnated silica gel, and aminopropyl silica gel were packed in a 190-mm long $1 / 16^{\prime \prime} \times 1 / 8^{\prime \prime}$ Teflon tube. The layers consisted of $64 \mathrm{mg}$ of anhydrous sodium sulfate, $170 \mathrm{mg}$ of oleum-impregnated silica gel, $64 \mathrm{mg}$ of anhydrous sodium sulfate, and $50 \mathrm{mg}$ of aminopropyl silica gel, from top to bottom, as shown in Fig. 2. To prevent reagent leakage, quartz wool was packed in the lowest layer of the capillary.

The dependence of the PCB recovery rates on the flow rates and the flow volumes was examined using the capillary multilayer column. A $50-\mu \mathrm{L}$ aliquot of PCB-contaminated oil $\left(0.54 \mathrm{mg} \mathrm{kg}^{-1}\right)$ was injected $10 \mathrm{~mm}$ from the fillers with a micropipette, to prevent broadening of the oil by capillary action in the fillers. After oil introduction, a hexane flow was applied. The solutions eluted with hexane were evaporated using a dryer. The PCBs in the evaporated eluate were then extracted into $50 \mu \mathrm{L}$ of DMSO by vortex mixing for $1 \mathrm{~min}$, and the resultant mixture was centrifuged at $5590 \mathrm{~g}$ for $1 \mathrm{~min}$. An $18-\mu \mathrm{L}$ sample 


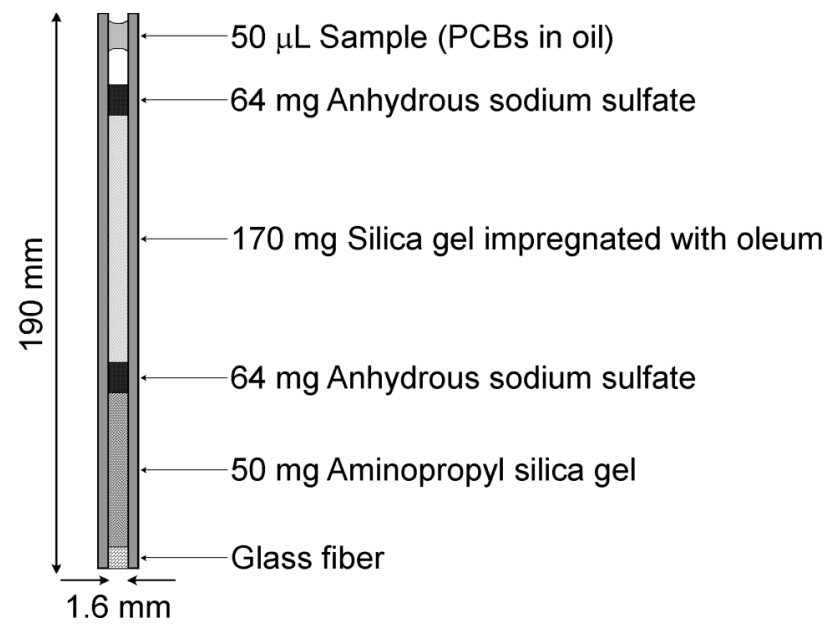

Fig. 2 Schematic diagram of the multilayer silica-gel column used for the cleanup of polychlorinated biphenyls (PCBs) in oil. Fillers were packed in a poly(tetrafluoroethylene) tube. PCB-contaminated oil $(50 \mu \mathrm{L})$ was injected into the column approximately $10 \mathrm{~mm}$ from the fillers using a micropipette.

of the lower DMSO phase was collected without the oil. Then, DMSO, PBS supplemented with $0.1 \%$ (w/w) N101, and antibody were added to obtain a total volume of $1.8 \mathrm{~mL}$. The PCBs in the collected DMSO were measured via a flow-based kinetic exclusion assay.

\section{Operating procedures}

The solutions were introduced via a microsyringe, and the flow rates were controlled using microsyringe pumps (KDS210; KD Scientific, Holliston, MA). A 1/4"-28 screw with 1/16" Tefzel tubing (Upchurch Scientific, Oak Harbor, WA) and a steel screw with a poly(ether ether ketone) (PEEK) tube $(0.5 \mathrm{~mm}$ diameter; Institute of Microchemical Technology, Kanagawa, Japan) were used to connect the syringes to aluminum blocks with ferrules. The column and Tefzel tube were connected using a PEEK union (Upchurch Scientific).

A $50-\mu \mathrm{L}$ aliquot of oil was introduced into the capillary column and eluted with hexane. The flow rates and flow volumes were manipulated soon after introducing the eluate from the capillary column into the microchip. After airflow to expunge hexane, the DMSO was expelled by flowing $1 \mathrm{~mL}$ of PBS. The obtained PBS solution was used as samples for an immunoassay.

\section{PCB immunoassay}

A flow-based kinetic exclusion assay instrument (KinExA 3000), obtained from Sapidyne Instruments Inc. (Boise, ID), was used for a PCB immunoassay. The immunoassay system was based on theory and operation that were previously described by us ${ }^{15-17}$ and others. ${ }^{18-20}$ The antigen-immobilized solid-phase, which was prepared as described previously, ${ }^{21}$ was captured in a small flow cell held at the focus of a fluorometer. The samples were prepared by mixing a pretreated solution with a detection antibody. The samples were flowed through the solid phase to capture a small fraction of free antibody on the solid phase. The amount of captured antibody on the solid phase was optically determined by labeling with a fluorescently labeled antibody. Samples without PCBs yield the highest response, and samples with very high concentrations of $\mathrm{PCB}$ lead to total inhibition of the specific binding signal. In this

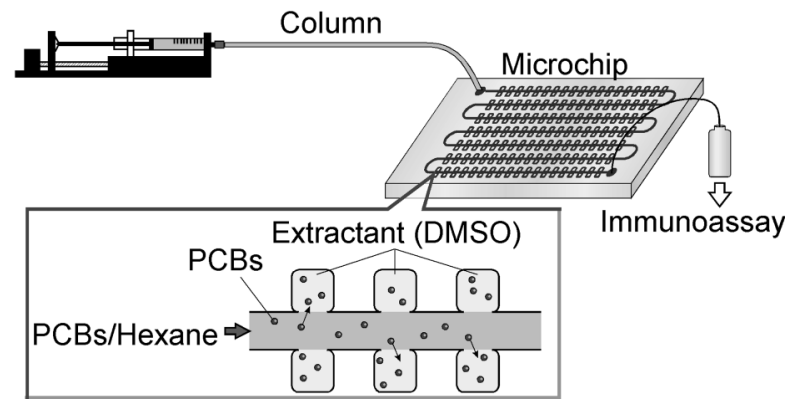

Fig. 3 Design of the multilayer silica-gel capillary column and microfluidic DMSO partitioning system used for pretreatment. The interfering substances in the PCB-contaminated oil are sulfonated and separated in the multilayer silica-gel capillary column. Any remaining interfering substances are cleaned up by extracting the PCBs in the eluate from the column into DMSO in the microchip.

work, the antibody concentration was $250 \mathrm{pM}$, and the measurement solutions were incubated at room temperature for a minimum of $30 \mathrm{~min}$. The concentration of DMSO in the measurement solution was $2 \%(\mathrm{v} / \mathrm{v})$. The blocking reagent, N101, was used to measure the PCBs.

\section{Visualization of streamlines}

Fluorescent microparticles were seeded in DMSO for the visualization of streamlines in the microrecesses. Fluorescence of the microparticles was observed using a fluorescence microscope.

\section{Investigation of enrichment factor}

PCBs in hexane were prepared by adding a commercial mixture of PCBs to pure hexane. A $300-\mu \mathrm{L}$ aliquot of $2.22 \mathrm{mg} \mathrm{L}^{-1} \mathrm{PCB}$ in a hexane solution was extracted into $300 \mu \mathrm{L}$ of pure DMSO by vortex mixing for $1 \mathrm{~min}$. The mixture was then centrifuged at $5590 \mathrm{~g}$ for $1 \mathrm{~min}$. A $170-\mu \mathrm{L}$ sample of the lower DMSO phase was collected without hexane. The PCB concentration in the collected DMSO was measured via a flow-based kinetic exclusion assay.

\section{Results and Discussion}

The overall design of the multilayer capillary column and the microchip used for the pretreatment is illustrated in Fig. 3. First, the interfering substances in PCB-contaminated oil were sulfonated and separated in the column using oleum and aminopropyl silica gels. Oleum sulfonates alkylbenzenes. The sulfonated alkylbenzenes and saturated hydrocarbons were separated using aminopropyl silica gel. Then, the PCBs were eluted with a small volume of hexane, to give a lower dilution of PCBs in the eluate. A high concentration of PCBs in the eluate was therefore obtained. The interfering substances that remained in the eluate were removed via hexane-DMSO concentrated extraction using a microchip; the relevant principles were reported previously. ${ }^{14}$ Although the eluate from the column is conventionally concentrated via evaporation, in our design, the PCBs in the eluate were directly extracted into DMSO without evaporation. The obtained samples were then analyzed using immunoassays. In order to realize the concept, a novel column that can elute PCBs at high concentrations and a microchip that can extract large amounts of PCBs are required.

Figure 4 shows the dependence of the PCB recovery rates on 


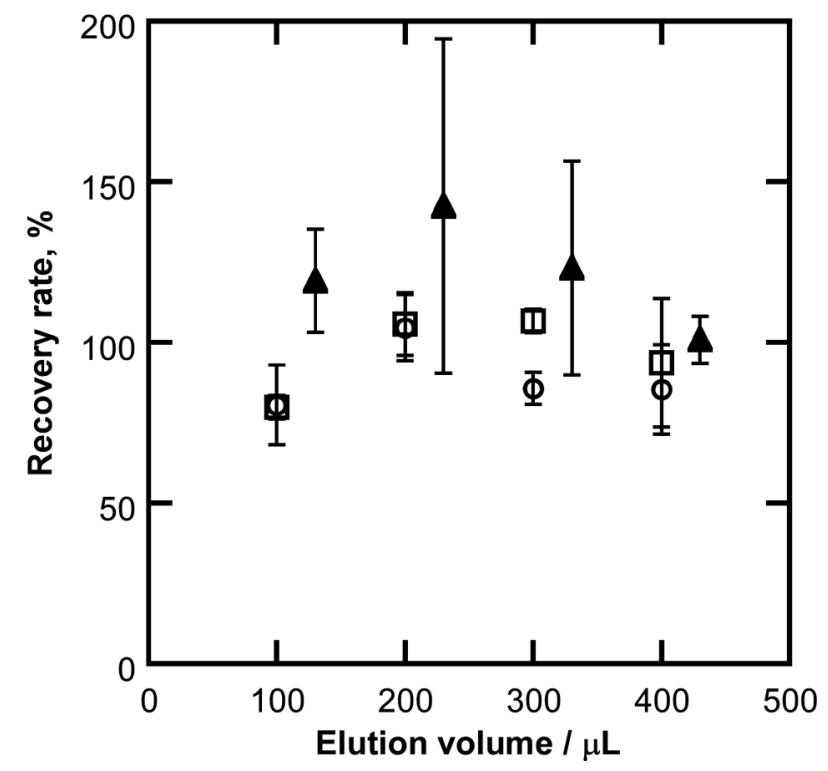

Fig. 4 Recovery rate of PCBs $\left(0.54 \mathrm{mg} \mathrm{kg}^{-1}\right)$ as a function of the hexane flow rates and the eluate volumes from the columns. $(\bigcirc),(\square)$, and $(\mathbf{\Lambda})$ represent the results for flow rates of 100,300 , and $500 \mu \mathrm{L} \mathrm{min}{ }^{-1}$, respectively. The error bars are \pm 1 standard deviation, based on triplicate measurements.

the hexane flow rates and eluate volumes from the columns. Recovery rates of almost $100 \%$ were achieved when the eluate volume was $200 \mu \mathrm{L}$ at flow rates of 100 and $300 \mu \mathrm{L} \mathrm{min}-1$. Recovery rates greater than $100 \%$ were measured when the flow rates were $500 \mu \mathrm{L} \mathrm{min}{ }^{-1}$, because there may be alkylbenzene contamination as a result of the short reaction time. Contamination by interfering substances occasionally occurred at a flow rate of $300 \mu \mathrm{L} \mathrm{min}$. We therefore used a flow rate of $100 \mu \mathrm{L} \mathrm{min}^{-1}$ for the column treatment. The initial 100 $200-\mu \mathrm{L}$ eluate fraction was partitioned with DMSO when the capillary column interfaced with the microchip. Since the column volume was $170 \mu \mathrm{L}$, the column processing time was completed within $2 \mathrm{~min}$, which is 3.5-times faster than the previous column processing time. ${ }^{13}$ Although $295 \mu \mathrm{L}$ of oil were eluted by $10 \mathrm{~mL}$ of hexane in the previous column (35-fold dilution), ${ }^{13} 50 \mu \mathrm{L}$ of oil were eluted by $200 \mu \mathrm{L}$ of hexane (fivefold dilution). The novel capillary column could therefore obtain PCBs in hexane at a dilution seven-times less than that obtained with the previous column. Furthermore, the amount of reagent required for the column was reduced to $1 / 12$ that required for the previous one. ${ }^{13}$

We previously investigated the enrichment factor in microfluidic DMSO partitioning between oil and DMSO. ${ }^{14}$ In the present design, the PCBs in hexane, corresponding to the eluate from the column, were extracted into DMSO via the microchip. The enrichment factor, $E$, between batch extraction and microflow extraction can be expressed as

$$
E=\left(C_{\text {Dmicro }}\right) / C_{\mathrm{D}}=(K+1) / K
$$

where $K$ is the apparent partition coefficient of the total PCB congeners; $C_{\mathrm{D}}$ and $C_{\text {Dmicro }}$ are the PCB concentrations in DMSO after batch extraction and after microfluidic extraction, respectively. ${ }^{14}$ A $300-\mu \mathrm{L}$ aliquot of $2.22 \mathrm{mg} \mathrm{L}^{-1}$ PCB in a hexane solution was extracted into $300 \mu \mathrm{L}$ of pure DMSO. A $170-\mu \mathrm{L}$ sample of the DMSO phase was collected without hexane. The PCB concentration in the collected DMSO was estimated to be $1.56 \pm 0.14 \mathrm{mg} \mathrm{L}^{-1}$ via a flow-based kinetic exclusion assay; the measurement errors corresponded to the standard deviation of the triplicate measurements. Therefore, $K$ and $E$ were calculated to be $0.42 \pm 0.10$ and $3.36 \pm 0.77$, respectively. Since $E$ for the previous microchip was 2.61, higher enrichment is expected. Furthermore, the improved microchip can extract a four-times larger amount of PCBs than that extracted by the previous microchip because the total volume of the improved microrecesses was four-times larger than that of the previous microrecesses.

Fluid motions for microfluidic DMSO partitioning were examined in a microchip with multiple microrecesses. After acetone was introduced and filled the main microchannel and multiple microrecesses, the acetone was replaced by DMSO with a dissolved fluorescein sodium salt. All of the microrecesses were filled with fluorescent DMSO without any air bubbles, as shown in Fig. 5(A). Hexane could only flow into the main microchannel, and DMSO remained in the multiple microrecesses, as shown in Fig. 5(B). After the hexane was purged by an airflow (Fig. 5(C)), the fluorescent DMSO flowed towards the outlet of the microchannel, by dissolving in PBS (Fig. 5(D)). The fabricated microchip could therefore be used for microfluidic DMSO partitioning. Fluorescent microparticles were seeded in DMSO for the visualization of streamlines in the microrecesses. Vortices were observed in the rectangular microrecesses as a result of the shear force at the interface between DMSO and hexane as shown in Fig. 5(E). This vortex flow can enhance the molecular transport of PCBs from hexane to DMSO through the liquid-liquid interface.

After the column conditions and fluid motions in the microchip were examined, the column was interfaced with the microchip. The efficiencies of DMSO partitioning and DMSO elution from the microrecesses influenced the immunoassay sensitivity. The important factors concerning the extraction efficiency were the flow rate and the flow volume of the eluate. Although a lower flow rate provided a sufficient contact time for molecular transport, a longer processing time was required. Therefore, the highest flow rate, which provided a sufficient contact time, was investigated. As described above, the initial $100-200-\mu \mathrm{L}$ fraction of the eluate was examined. A $50-\mu \mathrm{L}$ aliquot of oil with $0.89 \mathrm{mg} \mathrm{kg}^{-1}$ PCBs was introduced into the capillary column and eluted at a flow rate of $100 \mu \mathrm{L} \mathrm{min}{ }^{-1}$. The flow rates and the flow volumes were manipulated soon after introducing the eluate from the capillary column into the microchip; the flow rates were adjusted to 5,10 , and $15 \mu \mathrm{L} \mathrm{min}{ }^{-1}$, and the flow volumes were adjusted to 100,150 , and $200 \mu \mathrm{L}$. After airflow, the DMSO was expelled by flowing $1 \mathrm{~mL}$ of PBS at $500 \mu \mathrm{L} \mathrm{min}^{-1}$. Since the dead volumes in the main microchannel and tubes were estimated to be about $50 \mu \mathrm{L}$, $950 \mu \mathrm{L}$ of PBS could be obtained. Samples were prepared by adding PBS, N101, DMSO, and the antibody to obtain a total volume of $1.0 \mathrm{~mL}$. Figure 6 shows the results of the relative responses, with the error bars corresponding to the standard deviation of three replicates of the experiments. Lower relative responses indicate a higher concentration of PCBs in DMSO. Furthermore, a short process time is favorable. We therefore used a flow rate of $10 \mu \mathrm{L} \mathrm{min}$ m $^{-1}$ and a flow volume of $150 \mu \mathrm{L}$ for DMSO partitioning in our experiments.

Although DMSO can be completely eluted from the multiple microrecesses using a lower flow rate, a lower flow rate leads to a longer processing time. The highest flow rate that provides a sufficient contact time to completely elute DMSO was therefore investigated in a similar way to that described in our previous paper. ${ }^{14}$ Flow rates of less than $500 \mu \mathrm{L} \mathrm{min}{ }^{-1}$ provided minimum 


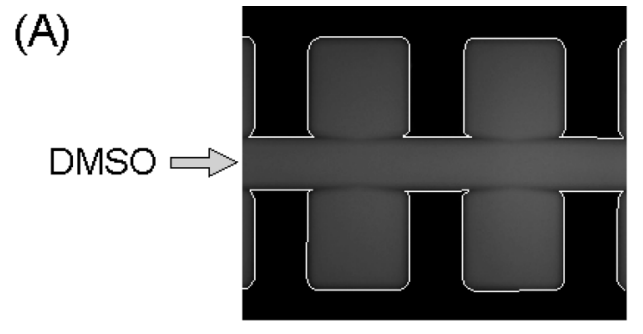

(B)

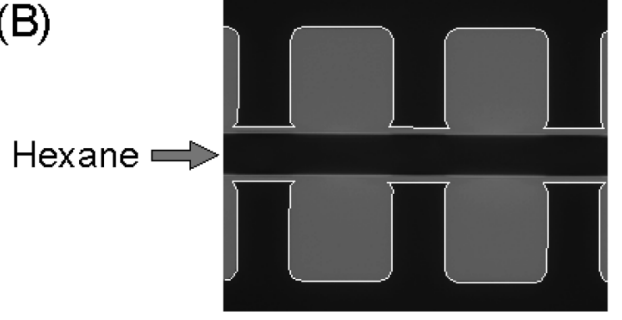

(C)

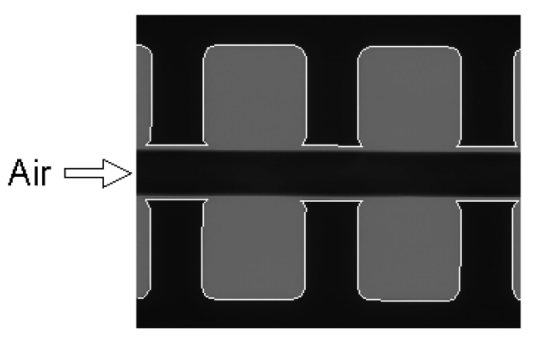

(D)

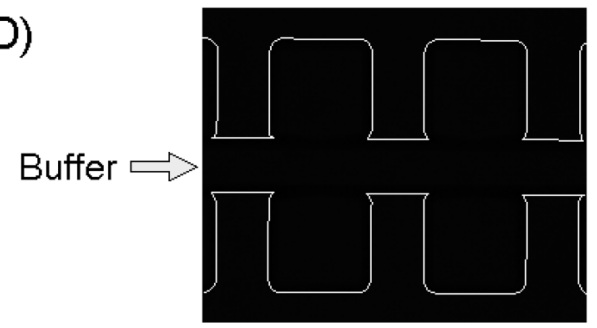

(E)

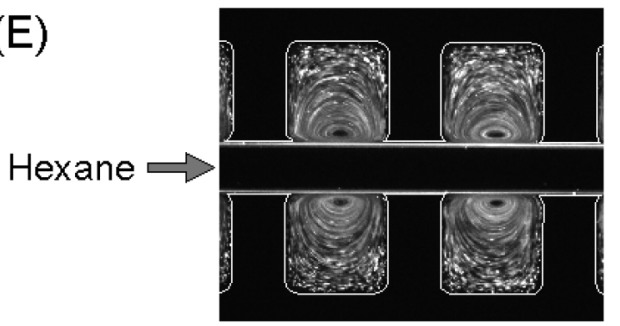

Fig. 5 Fluid motions in a microchip with multiple microrecesses. The gray areas correspond to fluorescent DMSO. (A) Microrecesses filled with DMSO without any air bubbles. (B) DMSO remained after hexane flow. (C) Only hexane was purged by the airflow. (D) DMSO (gray) was eluted with a buffer solution. (E) Streamlines in the microrecesses. Gray lines correspond to the motion of the fluorescent microparticles.

relative responses, indicating complete DMSO elution when $1 \mathrm{~mL}$ of PBS was flowed into the main microchannel. Consequently, we used a flow rate of $500 \mu \mathrm{L} \mathrm{min}^{-1}$ for the elution process in our experiments.

Once the conditions for the flow rates and flow volumes were determined, the concentration dependence of the flow-based immunoassay pretreated with the capillary column and the microchip was examined. Figure 7 shows the standard curve, with error bars, from three replicates of the experiment. The solid line in Fig. 7 corresponds to curve fits for the experimental

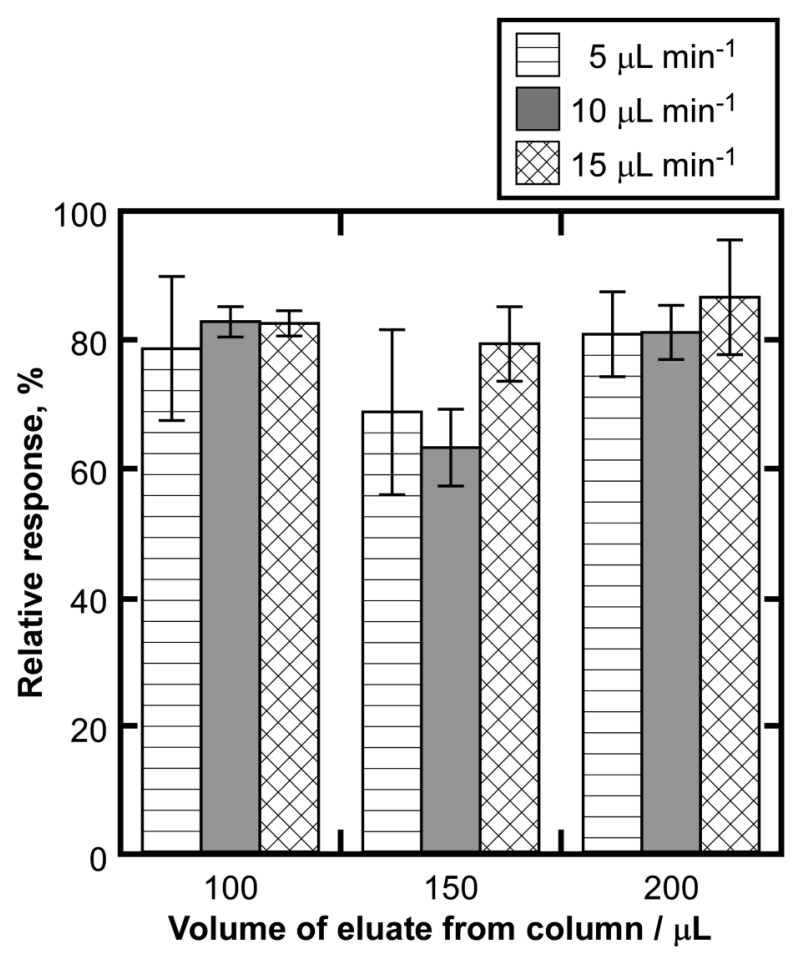

Fig. 6 DMSO partitioning efficiency as a function of the hexane flow rate. The error bars are \pm 1 standard deviation, based on triplicate measurements.

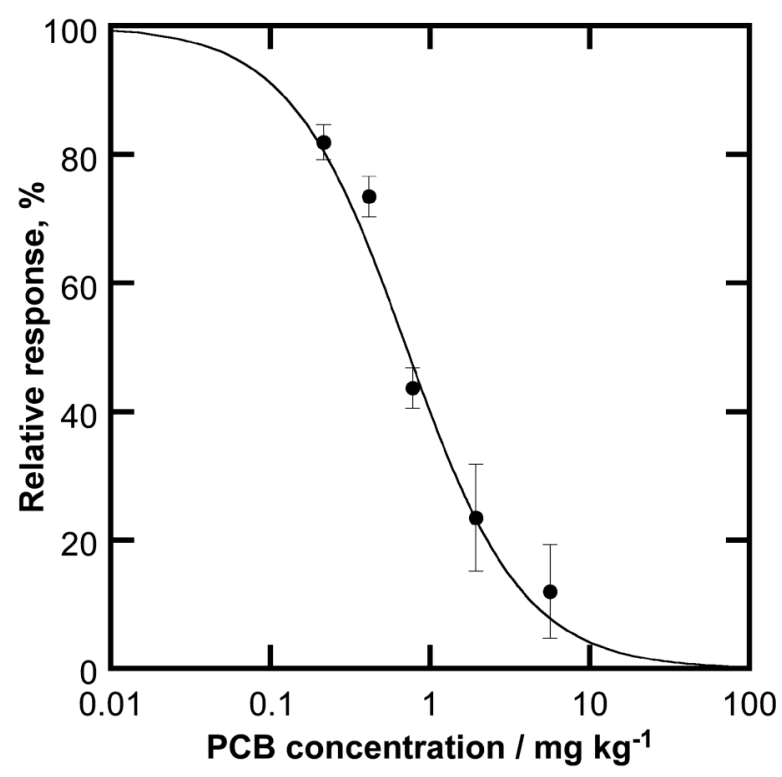

Fig. 7 Relative response as a function of the PCB concentration in oil. The solid line represents the curve fit to the experimental results. The error bars are \pm 1 standard deviation, based on triplicate measurements.

results obtained using the four-parameter logistic equation. ${ }^{14}$ The measurement range was estimated to be $0.22-2.27 \mathrm{mg} \mathrm{kg}^{-1}$, and was defined as the relative response from 20 to $80 \%$. The Japanese regulation criterion of $0.5 \mathrm{mg} \mathrm{kg}^{-1} 21$ could therefore be satisfied. The half-maximal inhibitory concentration was estimated to be $0.71 \mathrm{mg} \mathrm{kg}^{-1}$. The limit of detection, which was 
calculated based on the 3-sigma method, was determined to be $0.15 \mathrm{mg} \mathrm{kg}^{-1}$. The novel capillary column could obtain PCBs in hexane at a dilution seven-times less than that using the previous column. The enrichment factor in the developed method was 1.3-times larger than that using the previous microchip. The improved microchip can extract four-times larger amounts of PCBs than that obtained using the previous microchip. A 36-fold enrichment was therefore achieved in the present pretreatment compared with the previous one. However, 35-fold enrichment was achieved by evaporation in the previous pretreatment. The concentrations of the final sample solutions were therefore thought to be almost the same. A half-maximal inhibitory concentration of $0.71 \mathrm{mg} \mathrm{kg}^{-1}$ was achieved in the present method, compared with $0.38 \mathrm{mg} \mathrm{kg}^{-1}$ in the previous method.

In this work, a pretreatment method for PCBs in oil was developed using a continuous process involving a capillary column and a microchip with multiple microrecesses aligned along the microchannel. As a result of miniaturization of the column, the developed method can reduce the oil sample volume from $295 \mu \mathrm{L}$ for the previous method ${ }^{13}$ to $50 \mu \mathrm{L}$, and the reagent amount for the column can be reduced to $1 / 12$ of the previous amount. The miniaturization permitted less-diluted column operation as a result of efficient contact of the oil with oleum and prevention of diffusion of PCBs. By increasing the total volume of the microrecesses, larger amounts of PCBs could be extracted into the DMSO confined in the microrecesses. Since phase separation in the microchip can be achieved via the introduction of a solution or air, centrifugation is not required. Furthermore, the evaporation step for enrichment, which was required in the previous method, ${ }^{14}$ can be omitted as a result of combining a highly efficient capillary column and microchip for DMSO partitioning. The total pretreatment time for the newly developed method is only $20 \mathrm{~min}$, whereas that for the previous method was more than $1 \mathrm{~h}$, which included preparation of the evaporation instrument. Since the space required for pretreatment is small, our newly developed method could be a powerful tool for on-site PCB screening.

In this work, a mixture of PCBs (KC 300, 400, 500, and 600; 1:1:1:1 mixture) was used. However, real samples have different compositions of PCB congeners. The partition coefficients of PCBs between DMSO and hexane, and antibody reactivity, depend on the PCB congeners. The determination of PCBs in oil is therefore difficult. Since the previously developed immunoassay method using a multilayer column and DMSO partitioning can be used to screen PCBs in real oil samples, ${ }^{12}$ our method will be useful for on-site screening.

DMSO partitioning is typically used for dioxin and polycyclic aromatic hydrocarbons (PAHs) cleanup. Microfluidic DMSO partitioning will also be useful for these applications. Our method is also applicable to other liquid-liquid extractions, such as aqueous-organic extraction and aqueous two-phase extraction, and is useful for the separation of inorganic ion complexes and biomolecules, etc.

\section{Conclusions}

A rapid and facile pretreatment method for the immunoassay of PCBs in oil was developed. A multilayer capillary column, which reduces the amount of reagent required to less than 1/12 of that required in the previous method, was developed and evaluated. Recovery rates of nearly $100 \%$ from $200 \mu \mathrm{L}$ of eluate were achieved by the injection of $50-\mu \mathrm{L}$ oil samples. PCB concentrations seven-times higher than those using the previous column were eluted. A microchip for concentrated solvent extraction of PCBs in DMSO was developed. It could extract amounts of PCBs four-times larger than that extracted using the previous microchip. The multilayer capillary column was interfaced with a microchip in which PCBs were extracted into DMSO confined to multiple microrecesses. Pretreatment was completed within $20 \mathrm{~min}$, without the need for evaporation and centrifugation instruments. The sensitivity and measurement range were determined to be $0.15 \mathrm{mg} \mathrm{kg}^{-1}$ and 0.22 $2.27 \mathrm{mg} \mathrm{kg}^{-1}$, respectively, which satisfied the Japanese regulation criterion of $0.5 \mathrm{mg} \mathrm{kg}^{-1}$. The developed method will be useful as a simple, rapid, low-cost, on-site screening of the PCBs in oil. Furthermore, the integration of column and solvent extraction processes has been demonstrated for the first time.

\section{Acknowledgements}

We are grateful to Prof. Tomokazu Matsue of Tohoku University for assistance with microchip fabrication.

\section{References}

1. M. D. Erickson, "Analytical Chemistry of PCBs", 2nd ed., 1997, CRC Press, Boca Raton.

2. O. Hutzinger, S. Sage, and V. Zitko, "The Chemistry of PCB's', 1974, CRC Press, Boca Raton.

3. Stockholm Convention on Persistent Organic Pollutants Website, http://chm.pops.int/default.aspx.

4. J. Hong, T. Takahashi, T. Ishizaka, H. Toita, B. Min, and K. Honda, Environ. Eng. Res., 2011, 16, 11.

5. T. Sawatsubashi, C. Tsukahara, K. Baba, E. Ohi, A. Shinoda, and N. Miura, J. Chromatogr., A, 2008, 1177, 138.

6. M. Takada, H. Toda, and R. Uchida, Chemosphere, 2001, 3, 455.

7. M. R. Criado, I. R. Pereiro, and R. C. Torrijos, J. Chromatogr., A, 2004, 1056, 263.

8. N. Ohmura, T. R. Glass, K. Sasaki, T. Joh, Y. Taemi, and N. Yokobori, Bunseki Kagaku, 2006, 55, 1.

9. N. Ohmura, T. R. Glass, K. Sasaki, T. Joh, Y. Taemi, N. Yokobori, and S. Terakado, Bunseki Kagaku, 2006, 55, 341.

10. Y. Takagi, C. Kataoka, H. Kitagawa, N. Tateishi, K. Sawadaishi, and K. Honda, Organohalogen Compd., 2007, 69, 800 .

11. A. Okuyama, H. Takenaka, and H. Mizukami, Organohalogen Compd., 2007, 69, 804.

12. N. Ohmura, T. R. Glass, K. Sasaki, T. Joh, Y. Taemi, K. Imanishi, and S. Terakado, Bunseki Kagaku, 2006, 55, 519.

13. Japan, The Ministry of the Environmental Government, 2011, http://www.env.go.jp/press/file_view.php?serial=17471 \&hou_id=13760 (in Japanese).

14. A. Aota, Y. Date, S. Terakado, H. Sugiyama, and N. Ohmura, Anal. Chem., 2001, 83, 7834.

15. N. Ohmura, S. J. Lackie, and H. Saiki, Anal. Chem., 2001, 73,3392 .

16. N. Ohmura, Y. Tsukidate, H. Shinozaki, S. J. Lackie, and H. Saiki, Anal. Chem., 2003, 75, 104.

17. T. R. Glass, H. Saiki, D. A. Blake, R. C. Blake, II, S. J. Lackie, and N. Ohmura, Anal. Chem., 2004, 76, 767.

18. R. C. Blake, II, A. R. Pavlov, and D. A. Blake, Anal. Biochem., 1996, 272, 123.

19. D. A. Blake, P. Chakrabarti, M. Khosraviani, F. M. Hatcher, C. M. Westhoff, P. Goebel, D. E. Wylie, and R. C. Blake, II, J. Biol. Chem., 1996, 271, 27677. 
20. R. M. Carter, R. C. Blake, II, T. D. Nguyen, and L. A. Bostanian, Biosens. Bioelectron., 2003, 18, 69.

21. Japan, The Ministry of the Environmental Government,
2002, Regulations of Waste Management and Public Cleansing Law, 2002, http://www.env.go.jp/en/laws/recycle/ 03.pdf. 